\title{
Recomendaciones para la provisión de atención paliativa a personas con COVID-19
}

Jorge A. Ramos-Guerrero, * Ramiro López-Elizalde, Leticia Asencio-Huertas, Carlos Enríquez-López, Judith García de Alba-Verduzco, Oscar Flores-Munguía, Luis Hernández-Flores, Alberto León-Alatorre, Miguel Nakamura-López, Iris Narváez-Sarmiento, Juan Reyes-Torres, Patricia Ornelas-Tavares, Livier Ortiz-Coronado, Mónica Osio-Saldaña, Nayely Salazar-Trujillo, Alejandro Quiroz-Hernández, Dalia Vásquez-Vásquez y Bernardo Villa-Cornejo

Instituto de Seguridad y Servicios Sociales de los Trabajadores del Estado, Programa Institucional de Atención Paliativa, Ciudad de México, México

\section{Resumen}

La pandemia de infección por SARS-CoV-2, causante de COVID-19, ha afectado profundamente a los sistemas de salud y ha ocasionado un enorme impacto en las familias, las comunidades y las naciones. La estrategia de respuesta integral requiere que además de las consideraciones epidemiológicas, científicas y técnicas, no se olvide el sufrimiento humano asociado a la enfermedad, la vulnerabilidad y la muerte. La atención paliativa a personas con sospecha o diagnóstico de COVID-19 con evolución grave y sus familias debe ser también parte clave de la acción organizada que ayude al alivio del sufrimiento y mejore la calidad de vida mediante el control de los síntomas, el abordaje de las necesidades psicológicas, sociales y espirituales, el apoyo para la planificación de la atención avanzada y la articulación de los objetivos de la misma, el cuidado de la persona en la fase final de la vida, así como el soporte ante la toma de decisiones complejas y problemas éticos, entre otros. Se ofrecen recomendaciones para brindar los cuidados paliativos en el contexto de la pandemia de COVID-19.

PALABRAS CLAVE: COVID-19. Pandemia. Cuidados paliativos. Calidad de vida.

\section{Recommendations for the provision of palliative care to people with COVID-19}

\section{Abstract}

The pandemic of SARS-CoV-2 infection, which causes COVID-19, has deeply affected health systems and has had a significant impact on families, communities and nations. A comprehensive response strategy requires in addition to epidemiological, scientific and technical considerations, for human suffering associated with disease, vulnerability and death not to be forgotten. Palliative care for people with suspicion or diagnosis of COVID-19 with serious evolution and their families should also be a key part of organized actions that helps alleviate suffering and improve quality of life by controlling symptoms, addressing psychological, social and spiritual needs, support for advanced care and its goals, end-of-life care, as well as support in complex decision-making and ethical problems, among others. Recommendations are provided for offering palliative care in the COVID-19 pandemic context.

KEY WORDS: COVID-19. Pandemic. Palliative care. Quality of life. 


\section{Introducción}

En diciembre de 2019, en China aparecieron casos de neumonía que fueron causados por SARS-CoV-2 (como posteriormente se documentó), el cual es un nuevo coronavirus que genera COVID-19 (coronavirus disease-2019). El 11 de marzo de 2020, la Organización Mundial de la Salud declaró a esta enfermedad como pandemia global. Para el 12 de octubre del 2021, en el mundo se han reportado 236599025 casos de COVID-19 y 4831486 defunciones con una tasa de letalidad global de $2 \%$. En México, se confirmaron 3732429 casos y 282773 defunciones.

Esta emergencia sanitaria, como un tsunami, ha afectado profundamente a los sistemas de salud, generando un enorme impacto a las naciones, comunidades, familias y personas; ${ }^{2}$ a su vez, se ha constituido en un poderoso amplificador del sufrimiento, por lo que es imperativo "el alivio de la carga de dolor y angustia severa asociadas con las condiciones de salud que amenazan o limitan la vida, así como el final de la misma". ${ }^{3}$

La Organización Mundial de la Salud establece la recomendación de "brindar acceso a cuidados paliativos para COVID-19, prestando especial atención a la protección de las personas con afecciones de salud preexistentes, personas mayores y otras personas en riesgo". No brindar cuidados paliativos en estos contextos agravaría la tragedia de la pandemia y podría considerase un factor de fracaso de la atención médica. ${ }^{4-8}$

\section{Cuidados paliativos durante COVID-19}

Los cuidados paliativos incluyen "cuidados activos y holísticos, para personas de todas las edades, que experimentan sufrimiento grave relacionado con la salud, debido a una enfermedad severa, y especialmente de quienes se encuentran cerca del final de la vida. Su objetivo es mejorar la calidad de vida de los pacientes, sus familias y sus cuidadores". ${ }^{9}$ En el contexto actual, deben brindarse a personas con condiciones amenazantes o limitantes para la vida diferentes a COVID-19 (preexistentes o de inicio reciente); con infección respiratoria aguda grave (IRAG), con sospecha o confirmación de COVID-19, en particular a las personas a quienes no se les brinda soporte vital avanzado por cualquier motivo o con enfermedad severa con síntomas intensos, así como a quienes progresan a la fase final de la vida.

Se deben proveer y mantener disponibles cuidados activos para el control de los síntomas del paciente, el abordaje integral de las necesidades psicológicas, emocionales, sociales y espirituales; la planificación anticipada de la atención, el soporte en la toma de decisiones complejas, así como el apoyo a las familias a través de la comunicación asertiva y gestión de la incertidumbre ante la enfermedad. Es necesario coordinarse para la acción que permita otorgar cuidados paliativos a quien lo requiera. $3,4,6,10-12$

\section{Control de síntomas en el paciente con COVID-19 grave}

Muchos pacientes pueden deteriorarse rápidamente y experimentar síntomas intensos, por lo que es necesario contar con una estrategia, concisa y rápida para su manejo. $8,10-14$

- Disnea: se ha reportado hasta en $67 \%$ de los pacientes. En disnea severa, el medicamento de elección es la morfina, que al reducir la respuesta ventilatoria a hipercapnia e hipoxia disminuye el esfuerzo respiratorio y la sensación de asfixia, con lo que mejora la comodidad y la intensidad del síntoma se reduce entre 20 y $30 \%$. ${ }^{8,15-18}$

- Tos: está presente en 70 a 80 \% de los pacientes. En tos severa, el fármaco de elección es la morfina. ${ }^{15,19}$

- Angustia-ansiedad: se presenta en $43 \%$ de los pacientes. Es probable que se desencadene secundariamente a la disnea, el aislamiento social y el miedo ante la posibilidad de que su situación empeore y el desenlace sea fatal. ${ }^{16,20,21}$

- Delirium: se presenta en $24 \%$ de los pacientes. ${ }^{15,19}$ Se caracteriza por alteraciones en el nivel de conciencia y de la atención, asociado a alteraciones cognitivas y de la percepción. Clínicamente se expresa como hiperactivo (agitación, hipervigilancia y activación psicomotriz), hipoactivo (apatía, somnolencia, disminución de la atención y nivel de alerta) y mixto (alternancia de agitación con letargia). El diagnóstico es clínico y el tratamiento, en el caso de que sea posible, busca corregir las causas desencadenantes, que en COVID-19 generalmente son la hipoxemia, el estado inflamatorio agudo, fiebre 
Gaceta Médica de México. 2021;157

Tabla 1. Control de los síntomas severos en COVID-19

\begin{tabular}{|c|c|c|c|c|}
\hline \multirow{2}{*}{$\begin{array}{l}\text { Disnea } \\
\text { severa }\end{array}$} & \multicolumn{3}{|c|}{ Estrategias farmacológicas } & \multirow{2}{*}{$\begin{array}{l}\text { Estrategias no } \\
\text { farmacológicas }\end{array}$} \\
\hline & Fármaco & Dosis & Precauciones & \\
\hline & Morfina & $\begin{array}{l}\text {-VO: liberación rápida, } \\
5 \text { mg/4-6 horas. } \\
\text {-IV/SC: dosis inicial } 2.5 \mathrm{mg} / 4 \text { horas. } \\
\text {-Infusión continua (SC/V): } \\
10 \text { mg/24 horas ajustados } \\
\text { conforme respuesta. } \\
\text { Rescate con } 10 \% \text { de la dosis total } \\
\text { de morfina en } 24 \text { horas. }\end{array}$ & $\begin{array}{l}\text {-Vigilar neurotoxicidad, } \\
\text { alucinaciones, sedación intensa, } \\
\text { mioclonías, confusión. En este } \\
\text { caso, reducir la dosis en } 50 \text { \%. } \\
\text {-Utilizar antihemético en caso de } \\
\text { náusea o vómito. } \\
\text {-Agregar un laxante (picosulfato } \\
\text { de sodio, lactulosa o senósidos). } \\
\text {-Ante insuficiencia renal, calcular } \\
50 \% \text { de la dosis o utilizar } \\
\text { hidromorfona. }\end{array}$ & $\begin{array}{l}\text {-Medidas posturales: } \\
\text { posicionamiento con el } \\
\text { tórax en semifowler }>30^{\circ} \text {, } \\
\text { decúbito lateral o prono. } \\
\text { - Técnicas de relajación y } \\
\text { manejo de ansiedad. }\end{array}$ \\
\hline & Esteroides & $\begin{array}{l}\text {-Dexametasona (IV o SC), } 24 \text { mg/24 } \\
\text { horas. }\end{array}$ & -Continuar si están prescritos. & \\
\hline \multirow{2}{*}{$\begin{array}{l}\text { Tos } \\
\text { severa }\end{array}$} & Dextrometorfano & -VO: 15 a 30 mg/6 horas. & -120 mg/día como dosis máxima & \multirow{2}{*}{$\begin{array}{l}\text {-Higiene respiratoria. } \\
\text {-Humidificar el aire/oxígeno. } \\
\text {-Medidas posturales. }\end{array}$} \\
\hline & Morfina & $\begin{array}{l}\text {-VO: liberación rápida } \\
2.5 \text { mg/4-6 horas. } \\
\text {-IV/SC: dosis inicial } 1 \text { mg/4 horas. }\end{array}$ & $\begin{array}{l}\text {-Los pacientes con tos no } \\
\text { controlada o con abundantes } \\
\text { secreciones no deben recibir } \\
\text { medicación VO, transmucosa ni } \\
\text { sublingual. } \\
\text {-Valorar uso de cromoglicato de } \\
\text { sodio, } 10 \text { mg inhalados } \\
4 \text { veces/día. }\end{array}$ & \\
\hline \multirow[t]{2}{*}{ Ansiedad } & $\begin{array}{l}\text { Benzodiacepinas } \\
\text { (acción corta o } \\
\text { ultracorta) }\end{array}$ & $\begin{array}{l}\text {-Lorazepam (VO/SL/IV/SC), } \\
\text { 0.5-2 mg/6 horas (PRN). } \\
\text {-Midazolam (VO/SL/R/IV/SC), } \\
\text { 2.5-5 mg/6 horas (PRN). } \\
\text {-Clonazepam (VO), } \\
\text { 0.5-2 mg/6 horas (PRN). }\end{array}$ & $\begin{array}{l}\text {-Pueden precipitar un cuadro de } \\
\text { delirium. } \\
\text {-Deben utilizarse con precaución } \\
\text { en pacientes con retención de } \\
\text { dióxido de carbono. }\end{array}$ & \multirow{2}{*}{$\begin{array}{l}\text {-Relajación profunda, } \\
\text { respiración abdominal, } \\
\text { relajación muscular } \\
\text { progresiva, relajación en } \\
\text { imaginación e hipnosis. } \\
\text {-Técnicas cognitivas } \\
\text { de contención: dejar } \\
\text { pasar, aplazar y detener } \\
\text { pensamientos. } \\
\text {-Escucha activa y soporte } \\
\text { psicoemocional. }\end{array}$} \\
\hline & $\begin{array}{l}\text { Neurolépticos } \\
\text { con efecto } \\
\text { ansiolítico }\end{array}$ & $\begin{array}{l}\text {-Levomepromacina (SC/IV), } \\
7.5 \text { mg (hasta un máximo de tres } \\
\text { veces). } \\
\text {-Clorpromacina, } \\
\text { 4-6 mg/8-12 horas (VO) o 8-12 mg/ } \\
\text { día en perfusión (IV). }\end{array}$ & $\begin{array}{l}\text {-No se sugiere escalar. } \\
\text {-Útiles en caso de ansiedad y } \\
\text { delirio. }\end{array}$ & \\
\hline \multirow[t]{4}{*}{ Delirium } & Haloperidol & $\begin{array}{l}\text {-VO/SL/IM/IV/SC, } 1-5 \text { mg/4-6 horas. } \\
\text { Mantenimiento } 20 \text { mg/día. }\end{array}$ & $\begin{array}{l}\text { - En adultos, } 50 \text { mg/día como } \\
\text { dosis máxima. Vigilar intervalo } \\
\text { QT. }\end{array}$ & \multirow{4}{*}{$\begin{array}{l}\text {-Proporcionar un ambiente } \\
\text { tranquilo, evitar estímulos } \\
\text { excesivos como } \\
\text { temperatura extrema, luces } \\
\text { excesivas, ruido. } \\
\text {-Facilitar reorientación en } \\
\text { tiempo, espacio y personal. }\end{array}$} \\
\hline & Levomepromazina & -IV/SC: $12.5-25$ mg/dosis. & \multirow{2}{*}{$\begin{array}{l}\text { - Dosis máxima } 300 \text { mg/día. } \\
\text { - Segunda línea si se requiere } \\
\text { sedación. } \\
\text {-No se recomienda su uso SC por } \\
\text { ser irritante. }\end{array}$} & \\
\hline & Clorpromacina & -VO/SL/IVO: 0.5-1 mg/4-6 horas. & & \\
\hline & Benzodiacepinas & $\begin{array}{l}\text {-Lorazepam VO/SL/IV/SC, } \\
\text { 0.5-2 mg/6 horas. }\end{array}$ & $\begin{array}{l}\text {-Pueden exacerbar la agitación. } \\
\text { - Monitorizar continuamente. }\end{array}$ & \\
\hline
\end{tabular}

$\mathrm{IM}$ = intramuscular; IV = intravenoso, $\mathrm{PRN}=$ por razón necesaria, $\mathrm{SC}=$ subcutánea, $\mathrm{SL}=$ sublingual, $\mathrm{TM}=$ transmucoso, $\mathrm{VO}$ = vía oral; vía oral solo de encontrarse disponible, $\mathrm{R}=$ vía rectal. *Para su prescripción requieren la valoración por especialistas.

y las alteraciones hepáticas, además del tratamiento sintomático. ${ }^{22}$

El control farmacológico y no farmacológico de síntomas se describe en la Tabla 1.

\section{Sedación paliativa en COVID-19}

Se puede utilizar la sedación paliativa como maniobra terapéutica para disminuir el estado de conciencia 


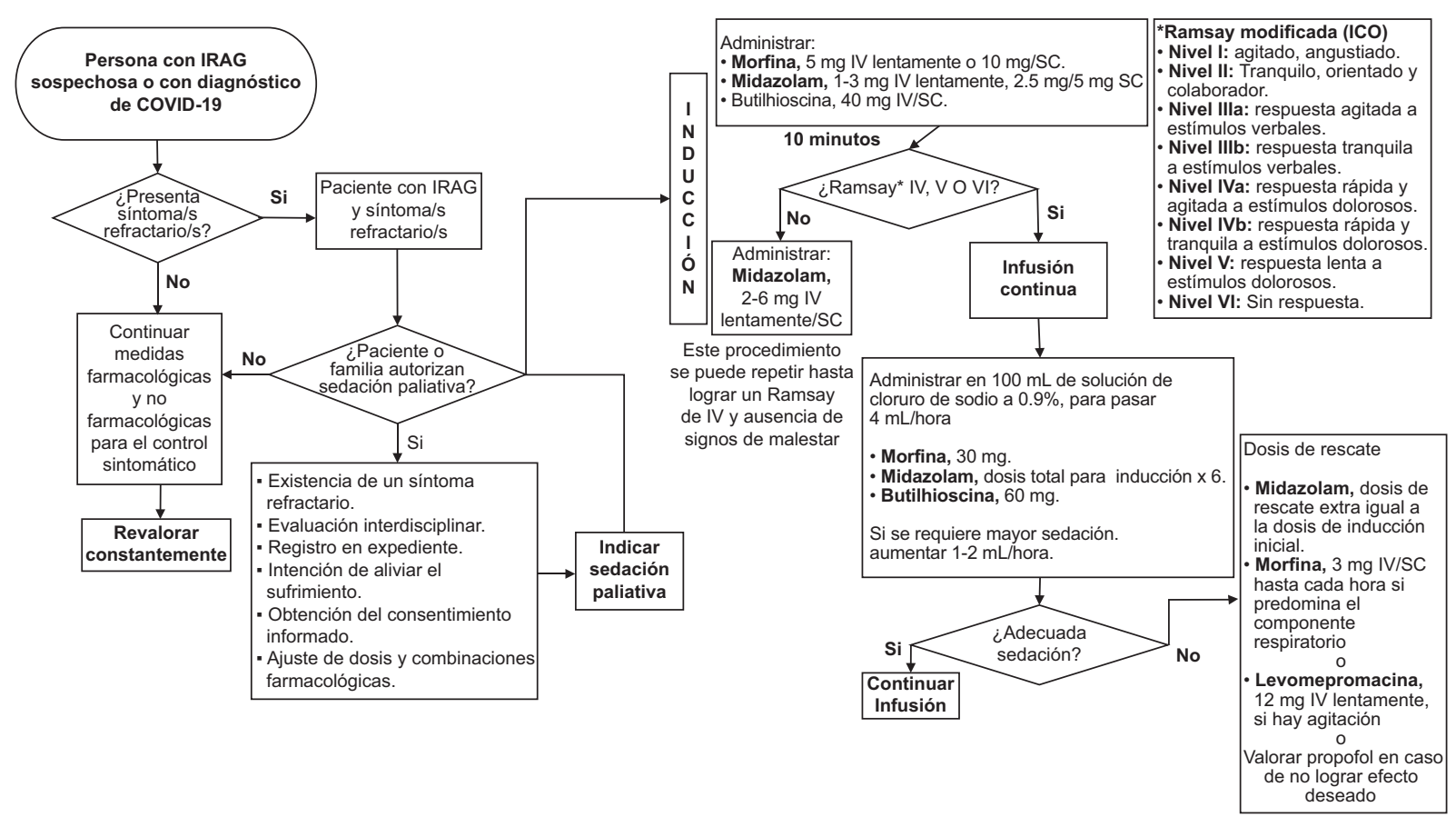

Figura 1. Procedimiento de sedación paliativa (adultos).

solo cuando uno o más síntomas severos sean refractarios, es decir, que no puedan ser controlados a pesar de esfuerzos terapéuticos en un tiempo razonable. ${ }^{21,23}$

En pacientes hipóxicos con progresión de la enfermedad que no reciben tratamiento intensivo y que evolucionan desfavorablemente con síntomas intensos (con frecuencia disnea, ansiedad y delirio), debe considerarse la sedación paliativa como una buena práctica clínica. ${ }^{4,15}$

Para que la sedación sea considerada ética y legalmente lícita se requiere:

- Presencia de un síntoma refractario.

- Evaluación interdisciplinar (para una decisión conjunta y consensuada).

- Registro en el expediente clínico.

- Intención de aliviar el sufrimiento (proporcionalidad terapéutica con el empleo de dosis mínimas eficaces para el síntoma que se desea aliviar).

- Obtención del consentimiento informado.

- Ajuste de las dosis y combinaciones farmacológicas conforme el nivel de conciencia requerido para el control (uso preferente de fármacos de rápida metabolización y vida media corta).

La sedación paliativa no debe confundirse con eutanasia. La muerte es una posibilidad secundaria al estado clínico de la persona. Si la indicación de administrar la sedación paliativa y el procedimiento aplicado ha sido correcta, la intención es el alivio del sufrimiento por la sintomatología refractaria y no provocar o acelerar la muerte. En la Figura 1 se propone un procedimiento de manejo. ${ }^{21,23}$

\section{Manejo paliativo en el domicilio}

Algunos pacientes deciden no ser atendidos en un hospital y prefieren permanecer en su casa. Esto plantea un desafío práctico derivado del escaso desarrollo de la atención domiciliaria en el país. Se enfrenta el reto de facilitar personal capacitado con adecuado equipo de protección personal y garantizar la prescripción de fármacos e insumos indispensables para el control sintomático. ${ }^{24-27}$

\section{Abordaje de la persona en la etapa final de la vida y el fallecimiento}

En los pacientes que fallecen por COVID-19, como principales causas de muerte se describen síndrome de insuficiencia respiratoria aguda (SIRA) (90\%), insuficiencia cardiaca aguda (60\%), insuficiencia renal aguda (18\%), choque (12\%) y coagulación intravascular diseminada $(6 \%) .{ }^{16} \mathrm{Al}$ entrar en la fase final de 
la vida es necesaria la adecuación del esfuerzo terapéutico, el cual deberá estar centrado en el compromiso de no abandono y en mantener el alivio y confort, además del retiro de medicamentos o medidas fútiles carentes de valor significativo para el bienestar.

En este tiempo, cientos de miles de personas han fallecido sin sentir el afecto y la cercanía de sus seres queridos. Las circunstancias hacen que sea imperativa la adopción de medidas muy estrictas para prevenir la transmisión de este virus; no obstante, también debemos reflexionar sobre el modo de facilitar un entorno más compasivo en el morir de estos pacientes, lo cual forma parte de la auténtica calidad asistencial. ${ }^{28,29}$

\section{Atención psicosocial y espiritual de la persona enferma y su familia}

Los aspectos de salud mental también requieren atención dinámica. La experiencia en epidemias ha mostrado que la ausencia de un sistema eficaz de salud mental y apoyo psicosocial aumenta los riesgos de angustia psicológica y la progresión de psicopatologías. La población con mayor riesgo de experimentar alteraciones en su salud mental es la más susceptible de complicaciones en caso de adquirir la enfermedad (con comorbilidades o adultos mayores), aquellas con trastornos preexistentes de salud mental, los familiares que experimentan duelo complicado y los profesionales de la salud. Se recomienda contar con profesionales de salud mental que aborden a pacientes, familias y equipos de atención, presencialmente o a través de tecnologías de la comunicación, y así identificar el malestar emocional, acompañar y, en su caso, realizar intervenciones psicosociales dirigidas a brindar soporte emocional, atención de necesidades espirituales y apoyo a las tareas del duelo. ${ }^{30-32}$

\section{Cuidados avanzados, voluntad anticipada y órdenes de no reanimación}

Por la evolución del cuadro clínico de COVID-19 no siempre es posible asegurar una discusión con el paciente y familia sobre sus deseos en torno al cuidado avanzado ante la progresión de la enfermedad; sin embargo, debe buscarse que este se centre en la persona y que, idealmente, sea abordado tempranamente. Las directrices anticipadas son cruciales para evitar un manejo intensivo de soporte vital (ventilación mecánica, cuidados intensivos, reanimación cardiopulmonar) cuando los pacientes no lo desean o no aporta beneficio para su calidad de vida. ${ }^{33,34} \mathrm{En}$ México, la Ley General de Salud otorga a los pacientes enfermos en situación terminal el derecho a "dar su consentimiento informado por escrito para la aplicación o no de tratamientos, medicamentos y cuidados paliativos adecuados a su enfermedad, necesidades y calidad de vida", así como a "renunciar, abandonar o negarse en cualquier momento a recibir o continuar el tratamiento que considere extraordinario". ${ }^{34}$

Existen situaciones clínicas en COVID-19 en las que no se debe utilizar un recurso por considerarse fútil (principio de beneficencia), ya que podría considerarse obstinación terapéutica al prolongar una muerte inminente (no maleficencia) y limitar el beneficio a otros pacientes con mayor posibilidad de respuesta (justicia). En consenso con la familia (y de ser posible con el paciente), el equipo tratante puede plantear una adecuación en la intensidad terapéutica y dirigir los esfuerzos hacia el confort y alivio sintomático. Ante la emergencia sanitaria es importante que se disponga de lineamientos claros con una perspectiva ética y de derechos humanos en torno al tema. Se debe evitar cualquier discriminación en el acceso a los recursos asistenciales por condiciones de edad, discapacidad u otra condición de vulnerabilidad. $28,29,35-39$

\section{Cuidado integral de los profesionales que atienden COVID-19}

Los equipos sanitarios en atención de primera línea y las áreas de apoyo experimentan una carga física y emocional que les hace particularmente vulnerables a experimentar trastornos físicos y mentales. Es necesario establecer estrategias que promuevan el cuidado integral, fortalezcan la resiliencia y detecten la necesidad de apoyo adicional tanto a nivel personal, de equipo y organizacional (Tabla 2). 8,24,40-44

\section{Organización para la acción}

Diversas estrategias han mostrado eficacia en otros escenarios críticos y pueden adaptarse a nivel nacional, institucional o local para asegurar la provisión de cuidados paliativos. Entre otras, se pueden señalar las siguientes: $6,41,45$

- Optimizar la coordinación entre los servicios involucrados en la atención de la persona con COVID-19 para que se brinde consulta directa o a distancia a los profesionales de "primera línea" 
Tabla 2. Cuidado integral de los profesionales que atienden COVID-19

\begin{tabular}{|c|c|}
\hline Organizacional & $\begin{array}{l}\text { 1. Proveer de adecuado y suficiente equipo de } \\
\text { protección personal e insumos para laborar de } \\
\text { acuerdo con el rol en la atención. } \\
\text { 2. Establecer protocolos de manejo clínico y uso } \\
\text { de equipo de protección personal para actuar } \\
\text { con más confianza y menos estrés. } \\
\text { 3. Reconocer a los profesionales y hacer } \\
\text { énfasis en el gran valor humano que tiene su } \\
\text { contribución. } \\
\text { 4. Generar estrategias de detección del burnout } \\
\text { y fatiga por compasión. } \\
\text { 5. Vinculación a grupos de apoyo emocional y } \\
\text { atención psicológica virtual o presencial. } \\
\text { 6. Establecer un comité monitor en salud mental } \\
\text { que promueva y supervise las medidas } \\
\text { establecidas. }\end{array}$ \\
\hline Equipo & $\begin{array}{l}\text { 1. Definir metas claras y consistentes en relación } \\
\text { con la atención de los pacientes. } \\
\text { 2. Favorecer las retroalimentaciones positivas } \\
\text { dentro del equipo. } \\
\text { 3. Tener reuniones cortas con el equipo entre las } \\
\text { crisis para discutir y supervisar las acciones y } \\
\text { procesos. } \\
\text { 4. Apoyarse de profesionales de la salud mental } \\
\text { para el cuidado. }\end{array}$ \\
\hline Individual & $\begin{array}{l}\text { 1. Fortalecer el autocuidado, gestion de salud } \\
\text { integral y resiliencia. } \\
\text { 2. Compartir los sentimientos, ansiedades y } \\
\text { preocupaciones con personas de confianza. } \\
\text { 3. Practicar el afrontamiento al estrés con } \\
\text { técnicas de atención plena (mindfulness) o } \\
\text { relajación. } \\
\text { 4. Tomar "tiempo fuera" y fomentar emociones } \\
\text { positivas (recuerdos positivos). } \\
\text { 5. Comunicación continua con personas } \\
\text { significativas. } \\
\text { 6. Reconocer cuándo se requiere ayuda y } \\
\text { solicitarla. }\end{array}$ \\
\hline
\end{tabular}

\section{Conclusión}

La integración de cuidados paliativos es esencial en la respuesta sanitaria ante las necesidades de los pacientes con COVID-19 y sus familias. Incorporar los principios del cuidado a través del control sintomático, el abordaje holístico de las necesidades de la persona, el acompañamiento en la toma de decisiones, el abordaje del duelo y el cuidado del equipo de salud permitirá disminuir el sufrimiento asociado a la enfermedad y la mejora de la calidad de vida, al mismo tiempo que se fomente el uso eficiente de los recursos asistenciales y el desarrollo de un sistema de salud de calidad.

\section{Agradecimientos}

A todo el personal de salud que con empatía y profesionalismo cuida a las personas con COVID-19. A la red de PALIATIVISSSTE por su compromiso humano.

\section{Conflicto de intereses}

Los autores declaran no tener conflicto de intereses alguno.

\section{Financiamiento}

La presente investigación no ha recibido ninguna beca específica de agencias de los sectores públicos, comercial o sin ánimo de lucro.

\section{Responsabilidades éticas}

Protección de personas y animales. Los autores en aquellos sitios donde se cuente con profesionales de cuidados paliativos y se capacite al personal en relación con el manejo terapéutico de los síntomas, el uso de opioides y bases de apoyo psicosocial donde se carezca de dichos expertos.

- Implementar protocolos de atención paliativa que permitan preservar la continuidad del cuidado integral con un enfoque centrado en las necesidad del paciente y su familia.

- Asegurar la disponibilidad de equipo de protección personal, opioides y demás insumos requeridos para la atención.

- Incorporar tecnologías de la comunicación como parte de la respuesta segura.

- Apoyarse en personal voluntariado capacitado. declaran que para esta investigación no se han realizado experimentos en seres humanos ni en animales.

Confidencialidad de los datos. Los autores declaran que en este artículo no aparecen datos de pacientes.

Derecho a la privacidad y consentimiento informado. Los autores declaran que en este artículo no aparecen datos de pacientes.

\section{Bibliografía}

1. Gobierno de México [Internet]. SA/SPPS/DGE/DIE/INDRE/UIES. Informe técnico diario COVID-19 México, 12/10/2021.

2. Redbrick L, Knaul FM, de Lima L, de Jonquiere C, Adelia A. The key role of palliative care in response to the COVID-19 tsunami of suffering. Lancet. 2020;395:1467-1469.

3. Canul FM, Farmer PE, Krakauer EL, de Lima L, Baudelio A, Jang Quete $X$, et al. Alleviating the access abyss in palliative care and pain relief-an imperative of universal health coverage: the Lancet Commission report. Lancet. 2018;391(10128):1391-1454. 
4. Arya A, Buchman S, Gagnon B, Downer J. Pandemic palliative care: beyond ventilators and saving lives. CMAJ. 2020;192:E400-E404.

5. Jouvet E, Sevaram M, Besançon K, Krishnaraj G, Hunt M, de Laat S, et al. Palliative care in humanitarian crises: a review of the literature. J International Humanitarian Action. 2018:3:3-5.

6. Etkind SN, Bone AE, Lovell N, Cripps RL, Harding R, Higginson IJ, et al. The Role and response of palliative care and hospice services in epidemics and pandemics: a rapid review to inform practice during the $\mathrm{CO}$ VID-19 pandemic. J Pain Symptom Manage. 2020;60:e31-e40.

7. World Health Organization [Internet]. Suiza: COVID-19 response: Seventy-Third World Health Assembly; 2020

8. Ting R, Edmonds P, Higginson IJ, Sleeman KE. Palliative care for patients with severe covid-19. BMJ. 2020;370:m2710.

9. Radbruch L, de Lima L, Knaul F, Wenk R, Ali Z, Bhatnaghar S, et al Redefining palliative care-a new consensus-based definition. J Pain Symptom Manage. 20200:754-764

10. Bowman BA, Esch AE, Back AL, Marshall N. Crisis symptom management and patient communication protocols are important tools for al clinicians responding to COVID-19. J Pain Symptom Manage. 2020;60:e98-e100.

11. Koh MYH. Palliative care in the time of COVID-19: reflections from the frontline. J Pain Symptom Manage. 2020;60:e3-e4.

12. Janssen DJA, Ekström M, Currow DC, Johnson MJ, Maddocks M, Simonds AK, et al. COVID-19: guidance on palliative care from a European Respiratory Society international task force. Eur Resp J. 2020;56:2002583.

13. Powell VD, Silveira MJ. What should palliative care's response be to the COVID-19 pandemic? J Pain Symptom Manage. 2020;60:e1-e3.

14. Fusi-Schdmidhauser T, Preston NJ, Keller N, Gamondi C. Conservative management of Covid-19 patients - emergency palliative care in action. J Pain Symptom Manage. 2020;60:e27.e30

15. Bajwah S, Wilcock A, Towers R, Costantini M, Bausewein C, Simon ST, et al. Managing the supportive care needs of those affected by $\mathrm{CO}$ VID-19. Eur Res J. 2020:55:2000815.

16. Keeley P, Buchanan D, Carolan C, Pivodic L, Tavabie S, Noble S Symptom burden and clinical profile of COVID-19 deaths: a rapid systematic review and evidence summary. BMJ Support Palliat Care. 2020;10:381-384

17. Schoenherr LA, Cook A, Peck S, Humphreys J, Goto Y, Saks NT, et al. Proactive identification of palliative care needs among patients with $\mathrm{CO}$ VID-19 in the ICU. J Pain Symptom Manage. 2020;60(3):e17-e21.

18. Adams $C$. Goals of care in a pandemic: our experience and recommendations. J Pain Symptom Manage. 2020;60:e15-e17

19. Lovell N, Maddocks M, Etkind SN, Taylor K, Carey I, Vora V et al. Characteristics, symptom management, and outcomes of 101 patients with COVID-19 referred for hospital palliative care. J Pain Symptom Manage. 2020;60:e77-e81

20. Salins N, Mani RK, Gursahani R, Simha N, Bhatnagar S. Symptom management and supportive care of serious COVID-19 patients and their families in India. Ind J Crit Care Med. 2020;24:435-444.

21. Universidad de Navarra [Internet]. España: Guías rápidas de apoyo y control sintomático en pacientes avanzados con COVID-19; 2020.

22. Kotfis K, Marra A, Wesley-Ely E. ICU delirium - a diagnostic and therapeutic challenge in the intensive care unit. Anaesthesiol Intensive Ther. 2018:50:160-167.

23. Arantzamendi M, Belar A, Payne S, Rijpstra M, Preston N, Menten J, et al. Clinical aspects of palliative sedation in prospective studies. A systematic review. J Pain Symptom Manage. 2020;61:831-844.

24. Gilissen J, Pivodic L, Unroe KT, van den-Block L. International COVID-19 palliative care guidance for nursing homes leaves key themes unaddressed. J Pain Symptom Manage. 2020;60:e56-e69.

25. Mercadante S, Adile C, Ferrera P, Giuliana F, Terruso L, Piccione T. Palliative care in the time of COVID-19. J Pain Symptom Manage. 2020;60:e79-e80.
26. Kuntz JG, Kavalieratos D, Esper GJ, Ogbu N, Mitchell J, McLean Ellis C. Feasibility and acceptability of inpatient palliative care E-family meetings during COVID-19 pandemic. J Pain Symptom Manage. 2020; 60:e28-e32.

27. Fausto J, Hirano L, Lam D, Mehta A, Mills B, Owens D, et al. Creating a palliative care inpatient response plan for COVID-19- The UW Medicine Experience. J Pain Symptom Manage. 2020;60:e21-e26.

28. De Montalvo-Jääskeläinen $\mathrm{F}$, Altisent-Trota $\mathrm{R}$, Bellver-Capella V, Cadena-Serrano $F$, de los Reyes-López M, de la Gándara-del Castillo Á, et al. Informe del Comité de Bioética de España sobre Aspectos Bioéticos de la priorización de recursos en el contexto de la crisis del coronavirus. Pers Bioet. 2020;24:1-13.

29. Guía de criterios éticos ante emergencias sanitarias en México en el contexto de la pandemia por COVID-19. México: Universidad de Anáhuac/Facultad de Bioética; 2020

30. Wallace CL, Wladkowski SP, Gibson A, White P. Grief during the COVID-19 pandemic: considerations for palliative care providers. J Pain Symptom Manage. 2020;60:e70-e76.

31. Bakar M, Capano E, Patterson M, Mclntyre B, Walsh CJ. The role of palliative care in caring for the families of patients with COVID-19. Am J Hosp Pal Med. 2020;37:866-868.

32. Ferrell BR, Handzo G, Picchi T, Puchalski $C$, Rosa WE. The urgency of spiritual care: COVID-19 and the critical need for whole-person palliation. J Pain Symptom Manage. 2020;60:e7-e11.

33. Petriceks AH, Schwartz AWN. Goals of care and COVID-19: A GOOD framework for dealing with uncertainty. Pall Support Care. 2020;18:379-381.

34. Ley General de Salud. México. Diario Oficial de la Federación 1984 Feb 07

35. Medina-Arellano M, Palacios-González C, Santos-Preciado ال Bioethics guide on scarce medical resource allocation in Mexico. Salud Publica Mex. 2020;62:1-3.

36. Wynne KJ, Petrova M, Coghlan R. Dying individuals and suffering populations: applying a population-level bioethics lens to palliative care in humanitarian contexts: before, during and after the COVID-19 pandemic. J Medical Ethics. 2020;46:1-12.

37. Rubio O, Estella A, Cabre L, Saralegui-Reta I, Martin MC, Zapata L, et al. Ethical recommendations for a difficult decision-making in intensive care units due to the exceptional situation of crisis by the COVID-19 pandemia: a rapid review and consensus of experts. Med Intensiva (Engl Ed). 20201;44:439-445

38. World Health Organization [Internet]. Suiza: Orientación ética sobre cuestiones planteadas por la pandemia del nuevo coronavirus (COVID-19), marzo 2020.

39. Toma de decisión para hospitalización o cuidados intensivos de personas mayores en el contexto de la pandemia de COVID-19. México: Instituto Nacional de Geriatría/Comisión Nacional de Bioética; 2020.

40. Fadul N, Elsayem AF, Bruera E. Integration of palliative care into COVID-19 pandemic planning. BMJ Sup Pall Care. 2021;11:40-44.

41. Feder SL, Akgün KM, Schulman-Green D. Palliative care strategies offer guidance to clinicians and comfort for COVID-19 patient and families. Heart Lung. 2020;49:227-228.

42. Shaukat N, Ali DM, Razzak J. Physical and mental health impacts of COVID-19 on healthcare workers: a scoping review. Int J Emer Med. 2020;13:40

43. Cabarkapa S, Nadjidai SE, Murgier J, Ng CH. The psychological impact of COVID-19 and other viral epidemics on frontline healthcare workers and ways to address it: a rapid systematic review. Brain Behav Immun Health. 2020;8:100144.

44. Heath C, Sommerfield A, von Ungern-Sternberg BS. Resilience strategies to manage psychological distress among healthcare workers during the COVID-19 pandemic: a narrative review. 2020;75:1364-1371.

45. Knights D, Knights F, Lawrie I. Upside down solutions: Palliative care and COVID-19. BMJ Support Palliat Care. 2020;bmjspcare-2020-002385. 